\title{
Laparoskopik cerrahide izoperistaltik intra-korporeal çift lineer stapler anastomoz: Erken oral beslenme; hızlı, kolay ve güvenilir teknik
}

\author{
Isoperistaltic intracorporeal double linear stapler anastomosis in laparascopic surgery: Early oral \\ nutrition; a fast, easy, and reliable technique
}

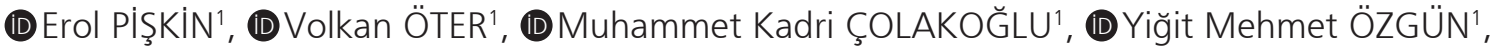 \\ (D) Osman AYDIN ${ }^{1}$, (DErdal Birol BOSTANCI²
}

\author{
Ankara Şehir Hastanesi Sağlık Uygulama ve Araştırma Merkezi, ' Gastroenteroloji Cerrahisi Kliniği, Ankara \\ Sağlık Bilimleri Üniversitesi, Ankara Şehir Hastanesi Sağlık Uygulama ve Araştırma Merkezi, ${ }^{2}$ Gastroenteroloji Cerrahisi Kliniği, Ankara
}

\begin{abstract}
Giriş ve Amaç: Gastrointestinal sistem kanser cerrahisinde minimal invaziv cerrahi; kısa dönem derlenme ve daha iyi fonksiyonel sonuçlarla birlikte, mide ve kolon kanseri cerrahisinde uzun dönem onkolojik sonuçlardan ödün vermeden uygulanmaktadır. Bu çalışmada amacımız laparoskopik radikal distal intrakorporeal Billroth II ve laparoskopik sağsol hemikolektomi sonrasında izoperistaltik ileokolik ve izoperistaltik kolokolik çift lineer stapler tekniği ile anastomoz yaptığımız hastaları incelemektir. Gereç ve Yöntem: Çalışmaya Aralık 2019- Mart 2021 tarihleri arasında kliniğimizde çift lineer stapler tekniği ile anastomoz yaptığımız 13 hasta dahil edildi. Tüm prosedürler total laparoskopik olarak gerçekleşti ve anastomozlar intrakorporeal olarak stapler yardımı ile tamamlandı. Bulgular: Tariflenen anastomozu uyguladığımız 13 hastanın mean yaş değeri $51.31 \pm 16.84$ idi. Hastaların 10'u (\%76.9) erkek ve 3'ü (\%23.1) kadın idi. Anastomozun median yapılış süresi $12.38 \pm 3.09$ dakika idi. Median ameliyat süresi 13 dakika (minimum-maksimum), (8-19 dakika) idi. Anastomoz esnasında ameliyat sonrası takiplerde 12 (\%92.3) hastada herhangi bir komplikasyon gelişmez iken 1 (7.7.\%) hastada anastomoz hattında kanama gözlendi. Kanama medikal takip ile tedavi edildi. Hiçbir hastaya relaparotomi gerekmedi. Sonuç: Laparoskopi sonrasında rekonstrüksiyon aşamasında bu stapler ile anastomoz tekniğinin hızlı ve kolay olarak uygulanabilir bir yöntem olduğu; anastomoz kaçağı açııından da güvenle uygulanabileceği görüşündeyiz.
\end{abstract}

Anahtar kelimeler: Laparoskopik cerrahi, izoperistaltik, stapler anastomoz, cerrahi teknik

\section{GíRiş ve AMAÇ}

Gastrointestinal sistem kanser cerrahisinde minimal invaziv cerrahi kısa dönem derlenme ve daha iyi fonksiyonel sonuçlarla birlikte; mide ve kolon kanseri cerrahisinde uzun dönem onkolojik sonuçlardan ödün vermeden uygulanmaktadır $(1,2)$.

\footnotetext{
İletişim: Erol PişKiN

Ankara Şehir Hastanesi Sağlık Uygulama ve Araştırma Merkezi, Gastroenteroloji Cerrahisi Kliniği, Bilkent Mahallesi, Ankara

E-mail: erol279@yahoo.com
}

Background and Aims: Minimally invasive surgery is associated with short-term recovery and better functional results in gastrointestinal system cancer surgery. It is applied in gastric and colon cancer surgery without compromising long-term oncological results. This study examines patients who underwent anastomosis with the double linear stapler technique with isoperistaltic intracorporeal ileocolic and isoperistaltic colocolic anastomosis after laparoscopic radical distal Billroth 2 and laparoscopic right-left hemicolectomy. Material and Method: Thirteen patients who underwent anastomosis with the double linear stapler technique in our clinic between December 2019 and March 2021 were included in this study. All procedures were performed entirely laparoscopically, and anastomoses were completed intracorporeally with the assistance of a stapler. Results: The mean age of 13 patients in whom we performed the described anastomosis was $51.31 \pm 16.84$ years. Ten (76.9\%) patients were male, and three $(23.1 \%)$ were female. The median duration of the anastomosis was $12.38 \pm 3.09$ minutes. The median operation time was 13 minutes (min-max) (8-19 minutes). While no perioperative complications developed in 12 (92.3\%) patients during anastomosis, perioperative intraluminal hemorrhage was observed in one $(7.7 \%)$ patient. This hemorrhage was treated medically with follow-up. No relaparotomy was required for any patient. Conclusion: This stapler anastomosis technique can be performed quickly and easily in the reconstruction phase after laparoscopy. We think it can be done safely concerning anastomotic leakage.

Key words: Laparoscopic surgery, isoperistaltic, stapler anastomosis, surgical technique

Malignite nedenli minimal invaziv hastalarda mobilizasyon ve lenfadenektomi sonrasında mini-laparotomi ile rekonstrüksiyon yapılabilmekte olup bu prosedüre laparoskopi yardımlı (LY) prosedür ve anastomoza ise ekstra-korporeal anastomoz denilmektedir. 
LY prosedürü yerine; total laparoskopik (TL), yani rekonstrüksiyon aşamasının da laparoskopik gerçekleştirilmesinin daha küçük cerrahi kesi izi, daha hızlı ameliyat sonrası derlenme ve anastomoz sırasında daha iyi görsellik elde etme gibi avantajları vardır $(3,4)$

Bu tip gerçekleştirilen TL prosedürlerde; anastomoz intra-korporeal olarak adlandırımaktadır.

Ekstra-korporeal anastomozun diğer bir dezavantajı ise spesmenin batın dışında traksiyonu gerekmesi ve daha ileri barsak mobilizasyonuna intiyaç duyulmasıdır.

Ayrıca kolektomi sonrasında intra-korporeal ve ekstra-korporeal anastomozlar karşılaştıııldığında; ameliyat sonrası anastomoz kaçağı insidansı, toplam morbidite ve 30-günlük mortalite açısından benzer sonuçlar elde edilmiştir (5).

Cerrahi sonrası hızlandırımış iyileşme (ERAS) protokolünün operatif komponentleri arasında nazogastrik tüp kullanılmaması, idrar sondasının erken çekilmesi, erken rejim başlanması gibi bileşenleri bulunmaktadır.

Bu protokolle birlikte laparoskopik cerrahi kombine edildiğinde; açık cerrahi ile aynı onkolojik sonuçlar elde edilebilmekte ve ameliyat sonrası komplikasyon oranı azalmaktadır $(6,7)$.

İntra-korporeal anastomozda stapler girişi için açılan gastrotomi, enterotomi ve kolotomi açıklı̆ııın kapatılması operasyon süresini uzatabilmektedir (8).

Kilitli sütür kullanımı ile anastomoz süresi önemli ölçüde kısaltılabilse de sütür kullanılmadan stapler ile kapatma daha da kısa sürede anastomoz sağlamaktadır (9).

Bu çalışmada amacımız laparoskopik radikal distal intra-korporeal Billroth 2 ve laparoskopik sağ-sol hemikolektomi sonrasında izoperistaltik ileokolik ve izoperistaltik kolokolik çift lineer stapler tekniği ile anastomoz yaptığımız hastaları incelemektir.

\section{GEREÇ ve YÖNTEM}

Bu retrospektif çalışma için Ankara Şehir Hastanesi Etik Kurulundan 07.04.2021 tarih ve E2-21-373 numarası ile etik kurul onayı alınmıştır.

Aralık 2019- Mart 2021 tarihleri arasında mide tümörü nedenli radikal distal gastrektomi yaptığımız 5 hasta, apendiks-çekum-çıkan kolon ve hepatik köşe tümörleri nedenli sağ hemikolektomi yaptığımız 7 hasta ve splenik köşe tümör nedenli sol hemikolektomi yağtığımız 1 hastayı çalışmamıza dahil ettik.
Tüm prosedürler total laparoskopik olarak gerçekleşti ve anastomozlar intra-korporeal olarak tamamlandı.

Distal gastrektomi sırasında D2 lenfadenektominin bir parçası olarak tüm hastalarda sol gastro-epiplokik arter ve ven kökü splenik hilus üzerinden kliplendi. Pedikül mideye doğru süpürüldü. Sonrasında mide büyük kurvaturda yaklaşık $8 \mathrm{~cm}$ segment iskeletize edilerek anastomoza hazırlandı. Yeterli mide arteryal beslenme-venöz drenaj için kısa gastrik arter-ven'ler korundu.

Daha önce inguinal herni nedenli laparoskopik ekstraperitoneal onarım (total extraperitoneal repair-TEP) operasyonu yapılan 1 hasta dışında tüm hastaların spesmenleri Pfannenstiel hattan çıkartıldı. Sol inguinal herni nedenli TEP ameliyatı yapılan hastanın ise patoloji spesmeni Mc-Burney insizyon ile çıkarıldı.

Tüm hastalarda makroskopik muayene ve patolojik frozen inceleme ile RO rezeksiyon marjı patolojik olarak doğrulandı. Treitz ligamanı'nın $30 \mathrm{~cm}$ distalinden jejunum antekolik olarak getirildi. Mide stapler hattına yakın olarak gastrotomi yapıldı. Jejunuma enterotomi sonrasında 60 mm lineer cutter (tercihen mor kartuş, yok ise mavi) ile gastrojejunal anastomoz yapıldı, anastomoz hattı kanama açısından izlendi. Bu işlemi takiben anastomoz aksına paralel olarak ön-arka hattan laparoskopik pensler ile kaldırıldı. Bu hat lineer cutter ile kapatılarak anastomoz tamamlandı (Resim 1). Çıkan dokuda gastrik ve jejunal duvar bütünlüğü görüldü. Kapatma hattında hemoraji olması durumunda laparoskopik metal klip ile kanama kontrolü sağlandı.

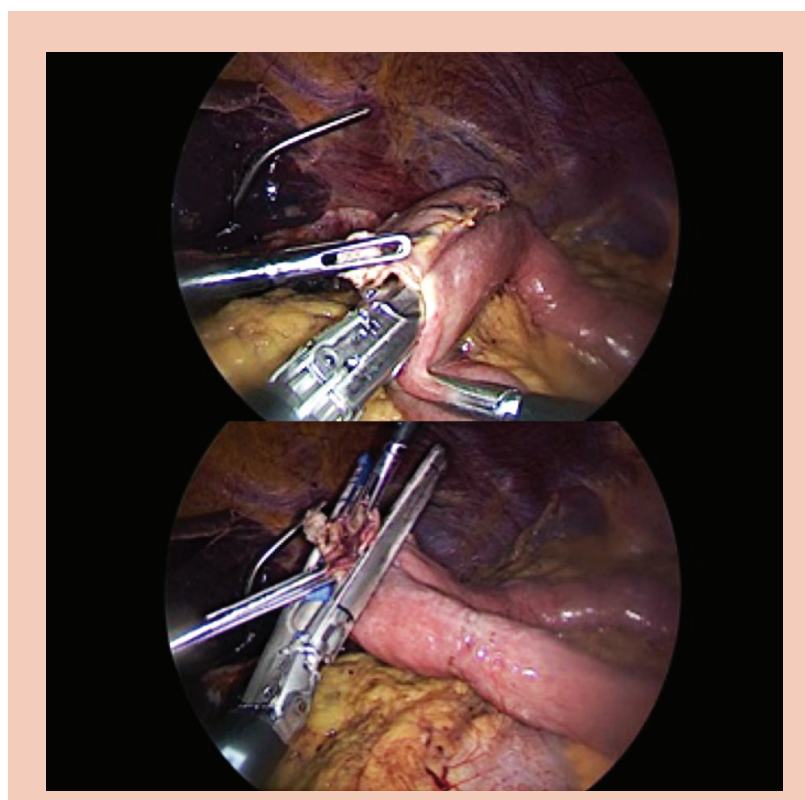

Resim 1. İzoperistaltik gastrojejunostomi anastomozunun yapılışı ve ortak kanalın stapler ile kapatılması. 
Sağ hemikolektomi rezeksiyon sonrasında ise ileum ve transvers kolon izoperistaltik olarak yan-yana gelecek yerleştirildi. Kolotomi ve enterotomi laparoskopik hook koter kesi modu kullanılarak mukozaya kadar dikkatli şekilde açıldı ve sonrasında laparoskopik disektör ile mukoza açıldı. 1 hastada 60 mm yeşil kartuş stapler ile anastomoz ve enterotomi kapatılması yapıldı. Diğer hastalarda ise $60 \mathrm{~mm}$ mavi lineer stapler (tercihen mor kartuş, yok ise mavi) yan-yana izoperistaltik anastomoz yapıldı. Açıklık anastomoz hattına dik olarak çekilerek yukarı kaldırıldı ve hat lineer stapler ile kapatıldı. Aynı prosedür sol hemikolektomi sonrasında da uygulandı (Resim 2).

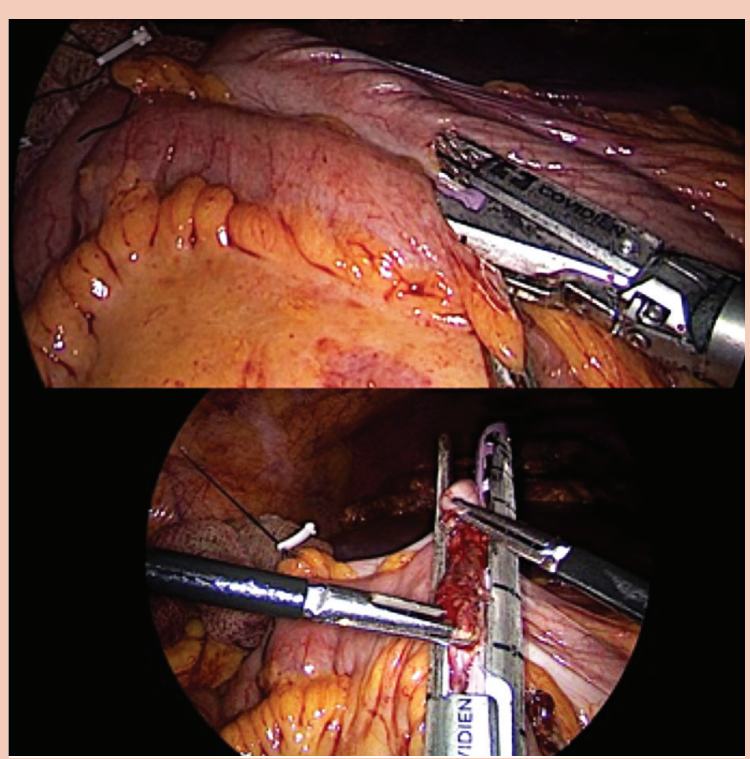

Resim 2. Illeotransversostomi anastomozunun yapılışı ve ortak kanalın stapler ile kapatılması.

Daha önce inguinal herni nedenli TEP operasyonu yapılan 1 hasta dışında tüm hastaların spesmenleri Pfannenstiel hattan çıkarıldı. TEP operasyonlu hastada ise mesh nedeni ile sağ Mc-Burney insizyondan çıkarıldı. Tüm hastalarda operasyon sonunda nazo-gastrik sondaları çekildi. Ameliyat sonrası 6 . saatte tüm hastaların rejimi su ile başlandı ve ameliyat sonrası 1. günde idrar sondası çekildi, sıvı gıdalar başlandı ve kademeli olarak arttırıldı.

\section{BULGULAR}

Tariflenen anastomozu uyguladığımız 13 hastanın ortalama yaş değeri $51.31 \pm 16.84$ idi. Hastaların 10'u (\%76.9) erkek ve 3'ü (\%23.1) kadın idi. Ameliyat öncesi değerlendirmede Amerikan Anestezi Derneği (American Society of Anaesthesiology- ASA) skoru kullanıldı. Hastaların 8'i (\%61.5) ASA I, 1 (\%7.7) hasta ASA II ve 4 (\%30.5) hasta ASA III idi. Hastaların ameliyat öncesi değerlendirmesinde; 5 (\%38.5) hastanın neoadjuvan kemoterapi almış lokal ileri evre mide adenokanseri olduğu, 2 (\%15.4) hastanın apendiks nöroendokrin tümörü, 1 (\%7.7) hastanın apendiks müsinöz adenokarsinomu olduğu, 2 (\%15.4) hastanın çekum tümörü, 1 (7.7\%) hastanın çıkan kolon tümörü, 1 (7.7\%) hastanın hepatik köşe tümörü ve 1 (7.7\%) hastanın da splenik köşe tümörü olduğu saptanmıştır. Hastaların stapler anastomozu için 10 (\%76.9) hastada mavi kartuş, 2'sinde (\%15.4) mor kartuş ve 1 'inde (\%7.7) ise yeşil kartuş tercih edilmiştir.

Anastomozun median yapılış süresi $12.38 \pm 3.09$ dakika idi. Median ameliyat süresi 13 dakika (minimum-maksimum) (8-19 dakika) idi. Anastomoz için 9 (\%69.2) hastada 2 adet kartuş ve 4 (\%30.8) hastada 3 adet stapler kartuşu anastomoz için yeterli olmuştur. Anastomoz esnasında perop 12 (\%92.3) hastada herhangi bir komplikasyon gelişmez iken 1 (\%7.7.) hastada takiplerinde anastomoz hattında kanama gözlenmiştir. Cerrahi spesmenler 12 (\%92.3) hastada Pfannenstiel insizyondan 1 (\%7.7) hastada Mc-Burney insizyondan çıkarılmıştır. Ameliyat sonrası sadece 1 (\%7.7.) hastada yara yeri enfeksiyonu gelişmiş olup sağ hemikolektomi yapılmış hastaların 3'ünde (\%23.1) şilöz fistül oluşmuş, 1 (\%7.7.) hastada ameliyat sonrası takiplerinde anastomoz hattında kanama nedenli melena gözlenmiş olup, medikal tedavi ile tamamen morbiditeler gerilemiş, hiçbir hastaya relaparotomi gerekmemiştir. Hastaların patoloji sonucunda 4 hasta T0, 1 hasta Tinsitu, 1 hastada T2 ve 7 hastada T3 olarak yorumlanmıştır. N evresi ise; 10 (\%76.9) hastada N0, 2 (\%15.4) hastada N1 ve 1 (\%7.7.) hastada N2 olarak saptanmıştır. Hastalardan çıkartılan toplam lenf nodlarının ortalama değeri $28.91 \pm 1.44$ ve ortanca değeri 24 lenf nodu (minimum-maksimum: 12-56 lenf nodu) olduğu gözlenmiştir.

\section{TARTIŞMA}

Mide ve kolon malignitesi nedenli laparoskopik rezeksiyon ve rekonstrüksiyon yaptığımı 13 hastada intra-korporeal çift lineer stapler anastomoz uygulanmış ve 1 hastamızda anastomoz hattından kanama dışında anastomoz ilişkili komplikasyon görülmemiştir. Bu hastada; stapler kartuşu yeşil renkli, stapler bacak boyu $3.4 \mathrm{~mm}$ ile anastomoz yapılmıştı, bu komplikasyon sonrasında tüm hastalarda mor veya mavi kartuş kullanıldı.

Ameliyatlarda anastomozları tamamlama süresi ortalama $12.38 \pm 3.09$ dakika idi.

Anastomoz sonrası stapler yerleştirilen açıklık sütürasyon ile de kapatılabilir fakat bu anastomoz süresini uzatmaktadır. Süre uzamasının negatif etkileri olarak; uzamış batın kontaminasyonu ve ortak kanaldaki mukozanın dışarı eversiyonu sonrasında kapatmanın zorlaşması sayılabilir. 
Laparoskopik mide cerrahisinde Delta-Shape olarak tanımlanan ve Billroth I anastomozu gerçekleştiren ilk makale Kanaya ve ark. tarafından yayınlanmıştır (10).

Literatürde laparoskopik kolon cerrahisi sırasında tariflediğimiz teknikle anastomoz tanımlayan makale ve hasta sayısı sınırlı olarak görülmektedir (11-13).

Jian-Cheng ve ark.ları çalışmalarında laparoskopik sağ hemikolektomi yapılan hastalarda intra-korporeal ve ekstra-korporeal anastomoz yapılan 85 hastayı analiz etmişler, intra-korporeal grupta daha az ameliyat sırasında kan kaybı, intestinal fonksiyonlarda daha kısa sürede geriye dönüş ve ameliyat sonrası daha az ağrı olduğunu belirtmişlerdir. Fakat bu çalışmada, TL grupta, ileum transeksiyonu anastomoz yapılması sırasında gerçekleştirilmiştir. Bizim sağ kolektomi vakalarımızda ileum transeksiyonu anastomoz yapılmadan önce gerçekleştirilmiş olup bu şekilde yapılan anastomoz ile karın içinin, barsak içeriği ile daha az kontaminasyonu olduğuna inanmaktayız (12).

Zhou ve ark. makalesinde laparoskopik kolektomi sonrası 20 hastaya çift lineer stapler anastomoz uygulamışlardır. Rejim açılması için ortalama 3 gün beklemişlerdir. Biz tüm hastalarımıza ameliyat sonrası aynı günde rejım başlayıp kademeli olarak arttırdık. Hastalarımızda ileus, anastomoz kaçağı gibi komplikasyonlar görülmedi (13).

Laparoskopik gastrektomi ve stapler anastomoz sonrasında afferent veya efferent anslarda darlık gelişebilmektedir (14). Bizim serimizde distal gastrektomi sonrasında hastalarımızda anastomoz darlığı görülmemiştir. Ayrıca hiçbir hastada pasaj problemi veya intraabdominal koleksiyon şüphesi nedenli radyolojik veya endoskopik görüntüleme yöntemlerine ihtiyaç duyulmamıştır.

Zhang ve ark. makalelerinde laparoskopik distal gastrektomi (LDG) sonrasında tüm hastalara endoskopi ile anastomoz kontrolü yaptıklarından bahsetmişlerdir. Bizim bu teknik ile anastomoz yaptığımız hastalarda ameliyat sırasında veya sonrasında endoskopik kontrol gerekmedi (15). Darlık gelişmemesi ve endoskopi veya radyolojik görüntüleme yöntemi ihtiyacı duyulmamasının nedeninin; enterotomi hattı traksiyonu sırasında anastomozun tamamen arkasında kalacak şekilde çekilmesi ve ortak kanaldan geniş doku çıkartılmayacak şekilde transeksiyon yapılmasının sonucu olduğu kanısındayız.

Ayrıca izoperistaltik anastomozun an-izoperistaltik anastomoza göre daha kısa mobilizasyon gerektirmesi ve barsak devamlılığının önceki barsak aksında olması nedeni ile daha fizyolojik olduğunu düşünmekteyiz.

Tanımladığımız anastomozda problem olarak stapler kesişme hatlarındaki zayıflık endişe verici olabilir fakat serimizde anastomoz kaçağı görülmemiştir.

Makalemizin zayıf yanları retrospektif olması, uzun dönem takip sonuçlarının olmaması ve laparoskopik koIon-mide cerrahisinin birlikte değerlendirilmiş olmasıdır.

Fakat kolon cerrahisi sonrasında; bu konfigürasyon ve ortak kanalın stapler ile kapatıldığı vaka ve makale sayısı sınırlıdır.

Serimizde anastomoz ile ilgili tek komplikasyon anastomoz hattı kanamasıdır ve bu da takip ile gerilemiştir.

Laparoskopi sonrasında rekonstrüksiyon aşamasında bu tekniğin hızlı ve kolay uygulanabilir bir yöntem olduğu; anastomoz kaçağı açısından da güvenle uygulanabileceği görüşündeyiz.

Etik Kurul: Bu çalışma için Ankara Şehir Hastanesi Etik Kurulundan 07.04.2021 tarih ve E2-21-373 numarası ile etik kurul onayı alınmıştır.

Çıkar Çatışması: Tüm yazarlar herhangi bir çıkar çatışması olmadığını kabul ederler.

\section{KAYNAKLAR}

1. Lu J, Yoon C, Xu B, et al. Long-term survival after minimally invasive versus open gastrectomy for gastric adenocarcinoma: A propensity sScore-matched analysis of patients in the United States and China. Ann Surg Oncol 2020;27:802-11.

2. Nishikawa T, Nozawa $H$, Kawai $K$, et al. Short- and long-term outcomes of minimally invasive versus open multivisceral resection for locally advanced colorectal cancer. Dis Colon Rectum 2019;62:40-6.

3. Lee SW, Tanigawa N, Nomura E, et al. Benefits of intracorporeal gastrointestinal anastomosis following laparoscopic distal gastrectomy. World J Surg Oncol 2012;10:267.

4. Ikeda O, Sakaguchi Y, Aoki Y, et al. Advantages of totally laparoscopic distal gastrectomy over laparoscopically assisted distal gastrectomy for gastric cancer. Surg Endosc 2009;23:2374-9.

5. Cirocchi R, Trastulli S, Farinella E, et al. Intracorporeal versus extracorporeal anastomosis during laparoscopic right hemicolectomy-systematic review and meta-analysis. Surg Oncol 2013;22:1-3.

6. Wang J, Lou Y, Wang Q, et al. Evaluation of the application of laparoscopy in enhanced recovery after surgery (ERAS) for gastric cancer: a Chinese multicenter analysis. Ann Transl Med 2020;8:543.

7. Tiefenthal M, Asklid D, Hjern F, Matthiessen P, Gustafsson UO. Laparoscopic and open right-sided colonic resection in daily routine practice. A prospective multicentre study within an Enhanced Recovery After Surgery (ERAS) protocol. Colorectal Dis 2016;18:187-94.

8. Milone M, Elmore U, Allaix ME, et al. Fashioning enterotomy closure after totally laparoscopic ileocolic anastomosis for right colon cancer: a multicenter experience. Surg Endosc 2020;34:557-63. 
9. Bracale U, Merola G, Cabras F, et al. The use of barbed suture for intracorporeal mechanical anastomosis during a totally laparoscopic right colectomy: Is it safe? A retrospective nonrandomized comparative multicenter study. Surg Innov 2018 25:267-73.

10. Kanaya S, Gomi T, Momoi H, et al. Delta-shaped anastomosis in totally laparoscopic Billroth I gastrectomy: new technique of intraabdominal gastroduodenostomy. J Am Coll Surg 2002;195:284-7.

11. Nepal P, Mori S, Kita Y, et al. Modified delta-shape anastomosis via the overlap method using linear staplers for colon cancer. J Anus Rectum Colon 2021;5:107-11.

12. Jian-Cheng $T$, Shu-Sheng $W$, Bo Z, Jian F, Liang Z. Total laparoscopic right hemicolectomy with 3-step stapled intracorporeal isoperistaltic ileocolic anastomosis for colon cancer: An evaluation of short-term outcomes. Medicine (Baltimore) 2016;95:e5538.
13. Zhou HT, Wang P, Liang JW, Su H, Zhou ZX. Short-term outcomes of overlapped delta-shaped anastomosis, an innovative intracorporeal anastomosis technique, in totally laparoscopic colectomy for colon cancer. World J Gastroenterol 2017;23:6726-32.

14. Junhyun L, Dongjin K, Wook K. Comparison of laparoscopy-assisted and totally laparoscopic Billroth-II distal gastrectomy for gastric cancer. J Korean Surg Soc 2012;82:135-42.

15. Zhang C, Xiao W, Chen K, et al. A new intracorporeal Billroth II stapled anastomosis technique in totally laparoscopic distal gastrectomy. Surg Endosc 2015;29:1636-42. 